\title{
What is culture? Culture is context-dependence!
}

\author{
Georg Northoff
}

Accepted: 22 July 2013/Published online: 16 August 2013

(C) Springer-Verlag Berlin Heidelberg 2013

\begin{abstract}
What is culture? The concept of culture has been defined in different ways and terms. I here take a different approach and determine the concept of culture as one specific instance the more general feature of context-dependence. The concept of context is here understood in a wider way that includes different kinds of contexts, social, cultural, mental, and bodily. Culture is then one specific instance of contextdependence. What exactly do I mean by the concept of 'dependence'? Following the philosopher Susan Hurely, there are different forms of context-dependence instrumental and non-instrumental. Instrumental dependence describes the indirect dependence for instance motor functions on the environment via the sensory functions. Non-instrumental context-dependence refers to a more direct dependence of the motor functions themselves on the context independent of the sensory functions. I now postulate that such non-instrumental context-dependence can also be observed in the brain that encodes its neural activity in direct dependence on its respective context. This is illustrated by three examples, reward, interoception, and social self. Such direct or better non-instrumental context-dependence of the brain's neural activity is possible only if the neural activity is encoded in a particular way in terms of differences between different stimuli rather than single stimuli themselves; this amounts to what I describe as difference-based coding as distinguished from stimulus-based coding. Does this entail cultural context-dependence? This is illustrated by a fourth example from depression as a psychiatric disorder. In conclusion, I here advocate the view that culture can be determined by direct or non-instrumental context-dependence which I postulate to be mediated by a particular encoding strategy of the brain, that is, difference-based coding.
\end{abstract}

G. Northoff $(\bowtie)$

Mind, Brain Imaging and Neuroethics Research Unit, Royal Ottawa Mental Health Centre, University of Ottawa Institute of Mental Health Research, 1145 Carling Avenue, Rm. 6435, Ottawa, ON K1Z 7K4, Canada

e-mail: georg.northoff@rohcg.on.ca; georg.northoff@theroyal.ca 
Keywords Culture $\cdot$ Context-dependence $\cdot$ Encoding $\cdot$ Difference-based coding $\cdot$ Stimulus-based coding

\section{Introduction}

What is culture? Various definitions have been put forward (see Han and Northoff 2008; Han et al. 2013 for a recent review). These definitions focus on how psychological functions and their underlying neural correlates differ in different cultural contexts. Due to its direct comparison between different cultures, cultural neuroscience is able to point out the sensitivity of both neural activity and associated psychological functions to different context thus entailing what can be described as context-dependence. The concept of context is here used in a specific way with respect to culture.

The definition of the concept of context by culture is though one only specific instance of a broader meaning of context. In addition to cultural context, we may also speak of social context that concerns the social environment including our social relationship with others. Moreover, we may also consider our own body and its vegetative functions as context, i.e., vegetative context. Depending on the viewpoint, our own self, taken in a phenomenological-experiential way, may also provide the context, a mental context if one wants to say so. This means that the meaning of context can also be understood in a rather wide way including different kinds of contexts, social, mental, vegetative, etc. Such broad meaning of context has to be distinguished from the more narrow definition of the concept of context that seems to be implicitly presupposed in cultural neuroscience.

The aim of this paper is to show that the brain's neural activity is necessarily and thus by default dependent on its context as used here in a wider definition. In short, I postulate that the brain's neural activity is intrinsically context-dependent. Such context-dependence is supposed to be mediated by a particular way of encoding neural activity in terms of difference rather than based on the single stimuli alone, i.e., difference-based coding rather than stimulus-based coding (See below for details). Cultural context-dependence is then regarded as one specific instance of the basic context-dependence of the brain's neural activity.

I here presuppose the wider concept of context as indicated above. Cultural contextdependence is then regarded a specific instance of context-dependence in general. The paper starts in a first section with a conceptual discussion of different forms of dependence, instrumental versus non-instrumental context-dependence. This will serve as conceptual basis for the subsequent discussion of context-dependence of the brain's neural activity. That will be exemplified by different example. I will discuss three examples from recent neuroscience, reward, interoception, and the social self to illustrate the noninstrumental context-dependence of the brain's neural activity. How about the specific instance of cultural context-dependence? That will be demonstrated by the example of a particular psychiatric disorder, depression, its symptoms and its underlying neural correlates. 


\section{Conceptual background IA: distinction between instrumental and non- instrumental dependence}

The philosopher Hurely (1998; 10, 341-343, 363-365) distinguishes between two different forms of dependence, instrumental and non-instrumental dependence; she illustrates that by the example of perceptual content and its relation to sensory input and motor output.

There is straightforward dependence of the perceptual content on the sensory input. For instance the kind of visual stimuli that form the sensory input determine our perceptual input. If the stimuli originate in a cat, our perceptual content will consist in a cat. Now however you move and shift your legs from the right to the left. Your perceptual content is now longer dominated by the cat but rather by the sofa on which she sits.

How do you explain the change in your perceptual content? You would assume that your movement and thus the motor output impacted the sensory input which in turn changed your perceptual content. This is what Susan Hurely describes as instrumental dependence: "For example, it is obvious that the perceptual content can vary with output that in turn has an effect on input: if I move around the corner I will see something I cannot see from where I am. This kind of dependence of perceptual content on output is merely instrumental. It operates via changes in input; changes in output are a means to changes in input." (Hurely 1998, 10).

There is yet another way though how the perceptual content can depend on the motor output. If for instance my eye muscles are paralyzed so that I cannot move my eyes and change my visual (and other sensory input). I may nevertheless have the visual experience that the world is shifting sideways. How is that possible? Instead of exerting an indirect impact on the perceptual content via the sensory input, the motor output may here directly modulate the perceptual content by itself.

In that case the motor output may directly change the perceptual input even if the sensory input remains the same. This is what Susan Hurely refers to as "noninstrumental dependence": "By contrast, when perceptual content depends noninstrumentally on output, it does not so via input, but directly. Perceptual distinctions vary with motor outputs even though inputs were fixed. If perceptual content depends noninstrumentally on output, then it is not a function of input." (Hurely 1998, 342; see also the various examples she discusses). Instrumental dependence can consequently be described by variation in all three, perceptual content as well as sensory and motor output. This is different in noninstrumental dependence where only the motor output and the perceptual content but not the sensory input varies.

\section{Conceptual background IB: from non-instrumental dependence to difference- based coding}

How is such noninstrumental dependence possible? Hurely argues that it is possible only when assuming that perception (and also action since she also makes the same case for intentions and their dependence on sensory and motor inputs) depends not necessarily on the sensory input (or the motor output in the case of intention) itself 
but rather on the relation between sensory input and motor output. Since the relation between sensory input and motor output changes in the case of isolated motor output variation (and sensory input constancy), perceptual content can be noninstrumentally dependent on the motor output.

Accordingly, the perceptual content (and the intention) is not based on the sensory input alone but rather on the relation between sensory input and motor output (see Hurely 1998: 214, 248, 342, 363, 371): "The various ways in which perceptions can depend noninstrumentally on output and intentions constrain one another. Not only do distinctions and invariants constrain one another, but perceptual content constrains the content of intentions and vice versa. The conclusion we approach is that in general both perceptual and intentional contents depend on relations between input and output, and hence are essentially interdependent." (see Hurely 1998, 371).

What does the dependence of the perceptual content on the relationship between perceptual input and motor output mean in neuronal terms? It means that the perceptual content must be encoded in the brain's neural activity in the difference between sensory and motor activity. Rather than being encoded in terms of sensory stimuli alone and thus in a stimulus-based way, the content is encoded as a difference, the sensory-motor difference, into neural activity. This amounts to difference-based coding as distinguished from stimulus-based coding as we will explain later in further detail.

How can we now link the assumption of non-instrumental dependence to context and the brain's neural activity? I postulate that difference-based coding makes possible the non-instrumental context-dependence of the brain's neural activity (with context understood in a wider sense). This will be exemplified by three examples from recent neuroscience, reward, interoception, and social self, in the following.

\section{Neuronal findings IA: reward and social context}

I here take reward and its dependence on social and vegetative context as paradigmatic example to illustrate how the brain and its neural activity deal with context. What about the neuronal mechanisms underlying reward and its assignment of value to exteroceptive stimuli? Reward value has been associated with neural activity in specific brain regions like the ventral striatum and the nucleus accumbens (VS/NACC), the ventromedial prefrontal cortex (VMPFC), and the midbrain with the ventral tegmental area (VTA) (Montague and Berns 2002; Montague et al. 2006; O'Doherty 2004; Schultz 2006; Glimcher 2011). The very same regions have also been associated with salience (Zink et al. 2003, 2004) and product preference (Knutson and Wimmer 2007; Menon 2011).

Moreover, the prediction error is quite well established in the neuroscientific literature on reward in both animals and humans (see Schultz 2006, 2007a, b; Montague and Berns 2002; Montague et al. 2006). Various studies demonstrate direct parametric dependence of the reward system's neural activity on the degree of the prediction error (see, for instance, O’Doherty 2011). 
The larger the prediction error, the stronger the neural activity changes in these regions of the reward circuitry during either a positive or negative prediction error, that is, reward or punishment. What is encoded into the reward circuitry's neural activity is thus not so much the single stimulus by itself, that is, the actual input, but its relation to the anticipated, that is, predicted, input, the prediction error.

Neural activity in reward circuitry, however, is not only determined by the relation between predicted input and actual input. In addition, the relation of the actual input to the social context may also need to be considered. The recently emerged discipline of neuroeconomics (see, for instance, Montague and King-Casas 2007; Fehr and Camerer 2007; Glimcher 2011; Engelmann and Hein 2013) presents particularly striking examples of how social inputs and thus the respective social context modulates neural and behavioral activity changes during reward.

Neuronal findings IB: reward-related neural activity is dependent on the social context

Let us give a paradigmatic example of an earlier functional magnetic resonance imaging (fMRI) study. Using fMRI, Fliessbach et al. (2007) demonstrated that the activity in reward circuitry (like the ventral striatum) was highest when the person in the scanner received $\$ 30$ in a gambling task and knew that another fictive player got less, that is, $\$ 10$. However, neural activity in reward circuitry decreased when the fictive player got $\$ 60$, even though the person in the scanner still received the same amount as before, that is, $\$ 30$.

How is this possible? One would expect the neural activity in the reward system to remain the same in both cases since the person receives the same amount of money, that is, $\$ 30$. But this is not the case. Neural activity increased when the person in the scanner received a higher amount than the person outside, whereas the opposite was the case in the reverse scenario.

Hence, neural activity in reward circuitry is determined not so much by the actual stimulus itself and its specifically associated value, that is, the $\$ 30$. Instead, neural activity seems to be rather determined by the relation between the actual stimulus, that is, the $\$ 30$, and the stimuli in the respective social context, that is, the other person receiving either $\$ 10$ or $\$ 60$.

Things become even more complicated when considering another example from neuroeconomics, the so-called endowment effect. The endowment effect describes the tendency to associate greater value with items that one owns when compared to those that one does not own. Knutson et al. (2008) showed that the medial prefrontal cortex exhibits greater neural activity changes during buying of a low-priced good when compared to the selling of the same good for the same price.

Since buying concerns the own person and ownership, the difference between selling and buying implies a difference in the socio-personal context concerning either the own or the other person. The results show that the medial prefrontal cortical neural activity changes are sensitive to ownership as manifest in their difference between selling and buying as related to own and other persons. Interestingly, the degree of neural activity in the insula even predicted the 
ownership, whether the item for selling was owned by the own or another person (Knutson et al. 2008).

Taken together, these (and other not mentioned here) results demonstrate that neural and behavioral effects of reward are very much dependent on the respective social and personal context and their respective stimuli.

Neuronal hypothesis IA: "social context-dependence" of neural activity

The first study demonstrated that neural activity in the reward system depended on whether the person inside the scanner receives a higher or lower amount of reward than the one outside the scanner. How is that possible? This is possible only when assuming that what is encoded into neural activity of reward is not the absolute amount of the actual stimulus itself, that is, the $\$ 30$, the person in the scanner receives by itself. Instead, the relation or better the difference between the two stimuli, that is, the difference in the amounts of money between the two persons inside and outside the scanner, must be encoded into the reward system's neural activity.

Both stimuli, i.e. the different amounts of money, are apparently compared and matched with each other with the result of this process determining the degree of neural activity in the reward system. This means that the social relation signified by the difference between the own and the other person's amounts of money is encoded into the neural activity change of the reward circuitry.

An analogous dependence on the respective context can be observed in the second study. Here, too, neural activity did not depend only on the stimulus itself, which was the same in both situations, that is, during buying and selling. Instead, the social and more specifically the personal context in which the person finds oneself whether she/he sells or buys, determines here the neural activity in, for instance, the medial prefrontal cortex and the insula.

Taken together, both examples (and many other examples from neuroeconomics) share the dependence of the behavioral and neural activities on the respective socialpersonal context. One may consequently speak of what I describe as "context dependence."

What do I means by the concept of context-dependence? Context dependence in our example means that both the reward value assigned to the stimulus and the reward circuitry's neural activity do not only depend on the single stimulus itself and its specific properties. Instead, both reward value and neural activity seem to depend on the stimulus's relation to other stimuli occurring at the same time in the respective social (and personal) environment, that is, another person also receiving reward or buying/selling. Since here it concerns the social environment, I speak of "social context dependence" (we will later touch upon other forms of context dependence).

Neuronal hypothesis IB: difference-based coding versus stimulus-based coding

How is it possible for such social context dependence to determine both behavioral and neural effects of reward? Let's discuss the exact underlying processes. My focus 
is here on the neural processes themselves independent of their psychological functions. What exactly happens neuronally? One exteroceptive stimulus, the one which is associated with the own person and the reward of $30 \$$ interacts with another exteroceptive stimulus, i.e., the one related to the other person and a reward of either $10 \$$ or $60 \$$.

The data show that the resulting neural activity does not only depend on the one exteroceptive stimulus alone, i.e., the one associated with the own person, but rather with the integral or difference between both exteroceptive stimuli. There must thus be some kind of yet unknown interaction between the two stimuli that allows to compute their difference or integral. Therefore I speak of extero-extero interaction that describes the postulated neural processes themselves independent of its psychological (phenomenal and mental) characterization. While one could easily rephrase what I here describe as extero-extero interaction in psychological terms, I nevertheless refrain from that in order to stay as closely as possible (at least in conceptual regard) to the neural processes or mechanisms themselves independent of their psychological and mental implications.

The data suggest that the postulated extero-extero interaction allows to encode the difference between the two exteroceptive stimuli in the neural activity of the reward system. Ne may thus want to speak of difference-based coding (see Northoff 2013a for details). Put in a nutshell, the concept of difference-based coding describes that the brain encodes differences between different stimuli rather than the stimuli themselves into neural activity. For example, the resulting neural activity in the reward system does neither correspond to the one exteroceptive stimulus alone and its $30 \$$ reward nor to the other exteroceptive stimuli that signals either $10 \$$ or $60 \$$ reward. Instead of being based on a single stimulus alone, i.e., stimulus-based coding, the encoded neural activity reflects the difference between the two exteroceptive stimuli entailing difference-based coding rather than stimulus-based coding.

How does such difference-based coding stand in relation to other forms of neural coding like predictive coding? The concept of predictive coding postulates that neural activity in particular regions like the ventral striatum (as for instance during reward) stems from the comparison between predicted and actual inputs (see Friston 1995, 1997, 2000, 2010; Montague et al. 2006). The measure or metric determining neural activity on a regional level thus pertains to a difference: predictive coding implies that the neural activity in particular regions is based on the encoding of a difference, the difference between predicted and actual input.

Without being able to go into details (see chapters 7-9 Northoff 2013a), predictive coding can be regarded a special instance of difference-based coding. Predictive coding applies to the prediction of an actual input by the preceding neural activity that is associated with a predicted input. The neural activity as related to the actual input, the stimulus-induced activity, is then supposed to result from the direct comparison and matching between predicted and actual inputs: the larger the difference between predicted and actual input, the larger the resulting stimulusinduced activity. The same now applies to difference-based coding which extends predictive coding beyond the relation between predicted and actual inputs to the relation between different stimuli in general including also different exteroceptive (and interoceptive as we well see below) stimuli. 
How is such difference-based coding related to the postulated social contextdependence? I suggest that the encoding of the difference between the actual stimulus, the $30 \$$ reward related to the own person, and the social stimulus, the $10 \$$ or $60 \$$ reward related to the other person, makes first and foremost possible the dependence of the brain's neural activity on its respective social context. Due to such difference-based coding, the brain's neural activity cannot avoid and is thus by default dependent on its respective social (and also cultural) context. I will later describe such direct and immediate context-dependence of the brain's neural activity as noninstrumental context-dependence. If, in contrast, the brain's neural activity were encoded in a stimulus-based rather than difference-based way, there would be no such direct and immediate social context-dependence.

\section{Neuronal hypothesis IC: non-instrumental context-dependence of neural activity during reward}

How can we apply the distinction between instrumental and noninstrumental dependence to our examples of social and vegetative context-dependence of reward? Does the social and vegetative context-dependence amount to instrumental or noninstrumental dependence?

Let us start with the first reward study that showed the degree of reward-related activity to be dependent on the relation between the own and others reward. There are three variables, the reward the person in the scanner receives, the reward the person outside the scanner receives, and the neural activity in the reward system of the person inside the scanner.

Presupposing instrumental dependence of the social context, one would now assume that the reward-related neural activity of the person inside the scanner depends directly on the amount of reward that person itself receives. In that case one would assume that the amount of money the person outside the scanner receives does not impact the degree of reward-related neural activity of the person inside the scanner. This means that the person inside the scanner should exhibit the same amount of neural activity when it receives $30 €$ independent of whether the person outside receives $10 €$ or $60 €$ (see Table 1 ).

The only way for the person outside the scanner to impact the neural activity of the person inside the scanner would here be to change the amount of reward the person inside the scanner receives. If for instance the person outside the scanner receives $60 €$ rather than $10 €$, it would imply that the person inside the scanner receives now longer $30 €$ but $10 €$.

That however was not the case as described in the data. Though the person in the scanner still received $30 €$ and thus exactly the same sum as before, it showed different degrees of neural activity when the reward of the person outside the scanner increased to $60 €$. Accordingly, the reward of the person inside the scanner thus did not change. What changed though the relation between the two persons' rewards by manipulating the degree of reward the person outside the scanner.

Most importantly, the change in the relation or difference between the two persons' rewards changed the degree of neural activity in the reward system of the 
Table 1 Instrumental versus noninstrumental context-dependence of neural activity during reward

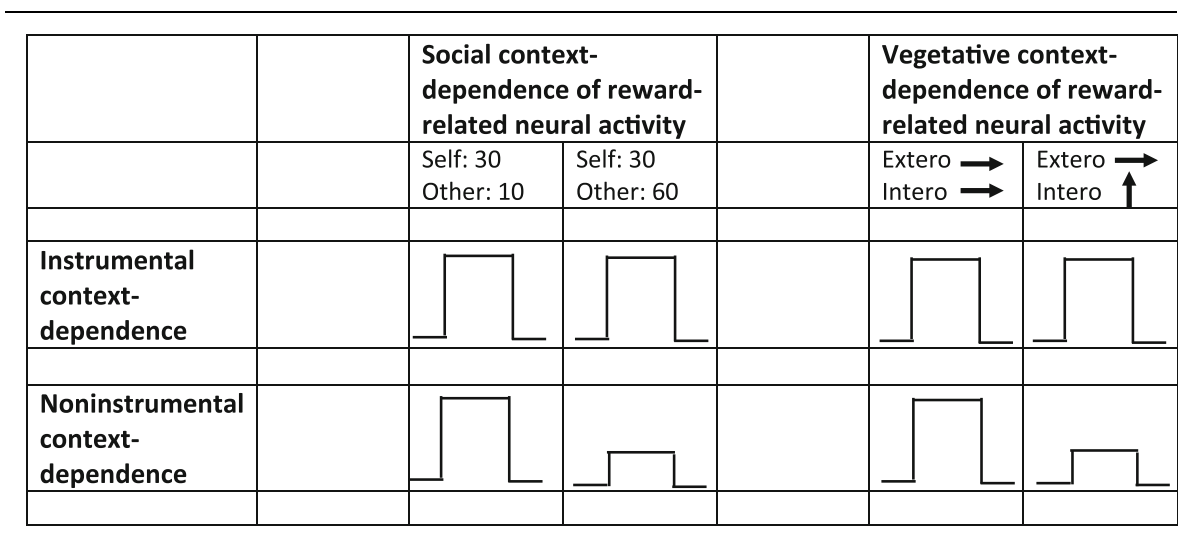

person inside the scanner (whose amount of reward did not change). This means that the reward-related activity is non instrumentally rather than merely instrumentally dependent on the social context.

Finally, it shall be pointed out that the concept of non-instrumental contextdependence does not imply mere quantitative changes. Instead, it implies qualitative changes. Why? The encoding of differences between stimuli is principally and thus qualitatively different from the encoding of the stimuli themselves. I thus postulate that we would have qualitatively different neural activity changes in the brain in the case of stimulus-based coding which would then also lead to principally different functions. Functions like reward as we understood currently would then probably no longer be present.

\section{Neuronal findings IIA: interoceptive stimuli from the body impact reward}

So far, I have considered the social context and how different exteroceptive stimuli are encoded into neural activity during reward via difference-based coding. However, there may be more to reward than only exteroceptive stimuli from the social context. The exteroceptive target stimuli are confronted in the brain not only with other exteroceptive stimuli from the respective social context but also with the stimuli that originate from the own body, the interoceptive stimuli.

There are many studies on reward and interoception, especially in the context of food, taste, hunger, appetite, and obesity (see, for instance, Rolls 2011 for an excellent review). Hunger and appetite obviously presuppose the involvement of the own body state and thus of interoceptive stimuli.

Montague argues in a series of papers that the temporal difference prediction error (TD-PD, which describes the difference between the predicted and actually occurring stimulus value; see Fiorillo et al. 2008; Schultz 2007a, b) model of reward neglects the input from the organism itself (i.e., its internal stimuli as manifest in interoceptive stimuli; Montague 2007a, b; Montague and King-Casas 2007; Rangel 
2008). This is even more relevant when considering the fact that the value assigned to the external stimulus may very much depend on the organism's internal state and thus on its interoceptive stimuli (Krebs et al. 1978).

How can further illustrate such dependence of reward on the interoceptive state of the body? For instance, a particular exteroceptive stimuli associated with cold water may be rewarding only if it is extremely hot and the organism itself is very hot, showing high temperature. If, in contrast, the organism is rather cold, showing low temperature, cold water may not be regarded as a rewarding stimulus and thus be assigned a rather low value (see Montague and King-Casas 2007 for this example).

This means that the same exteroceptive stimulus, the cold water, may be assigned a different value in the context of different bodily states and consecutively different interoceptive stimuli. Hence, there must be some kind of intero-extero interaction during the assignment of value and thus reward. This is indeed empirically supported by studies showing differences of reward in the presence of different electrolyte concentration or delivery of food (see for instance Kirk et al. 2012; Northoff et al. 2011).

\section{Neuronal findings IIB: subcortical regions mediate the impact of interoceptive stimuli on reward}

How is such interaction between intero- and exteroceptive stimuli mediated neuronally? Intero-extero interaction may be mediated specifically by subcortical regions. These subcortical regions include the PAG, the tectum, the colliculi, the hypothalamus, and the VTA that all show extensive convergence between interoand exteroceptive afferences (see part VIII in volume II for a more detailed description of subcortical regions).

In addition to the subcortical regions, cortical regions like the insula are also central. The insula has been implicated in the processing of interoceptive stimuli from one's body and subsequent bodily awareness (see Craig 2003, 2004; Wiebking et al. 2010). This goes well with the insula's connectivity pattern that is characterized by afferents from both intero- and exteroceptive sensory modalities. Interestingly, the insula has also been shown to be recruited during especially social reward tasks (see Montague and King-Casas 2007; Kirk et al. 2012; Naqvi and Bechara 2009, 2010).

The subcortical regions and the insula, including the latter's close connection to the supragenual anterior cingulate cortex (SACC; see also Craig 2010), are considered core regions of what is described as the "salience system" (see Menon 2011 for a recent summary). The salience system is supposed to be central in registering the need of the organism to dedicate and reallocate resources on the basis of its own states and the relevance, that is, salience, of the stimuli for itself given its own present bodily state.

The salience system is distinguished from the default-mode network (that includes midline regions), and the central executive network and its predominantly lateral cortical regions (see Menon 2011). Interestingly, many of the regions 
included in the salience system, especially insula, amygdala, SACC, and VTA, are also recruited during reward, thus suggesting strong overlap (some authors also propose identity between both) between reward and salience.

Taken together, the findings suggest involvement of interoceptive stimuli in reward. This is evidenced by the well established dependence of reward value on the body's interoceptive, that is, vegetative functions. Such assumption is further supported by the involvement of the regions of the salience system that mediates interoceptive and thus vegetative functions.

\section{Neuronal hypothesis IIA: "vegetative context-dependence" and the "framing effect"}

The findings described here underline the relevance of the body for reward. The body and its interoceptive stimuli may thus be regarded as yet another context that needs to be considered. More specifically, it is the vegetative state of the body as signified by its interoceptive stimuli that strongly impacts reward. One may consequently want to speak of a bodily or "vegetative context dependence" as distinguished from the "social context dependence" described earlier.

The concept of vegetative context-dependence describes the dependence of the neural activity during exteroceptive stimuli on the interoceptive stimuli from the own body. The data suggest that what is encoded into neural activity is not based on the single exteroceptive stimulus alone but also on its relation to the interoceptive stimuli from the own body. What is computed and thus encoded into neural activity is the integral or difference between intero- and exteroceptive stimuli. As in the case of extero-extero interaction (see above), one would thus want to speak of interoextero interaction that implies difference- rather than stimulus-based coding. I now postulate that such difference-based coding applies not only to the relation between different exteroceptive stimuli, i.e., extero-extero interaction, but also to the relation between intero- and exteroceptive stimuli, i.e., intero-extero interaction (see below for details).

The concept of vegetative context dependence comes close to what Martin Paulus (2007) calls "framing effects." Paulus (2007) proposes the body's interoceptive stimuli and thus its homeostasis to be central in reward and decision making. The value to the stimulus is not only assigned on a purely rational, for example, cognitive, basis but also by considering its value for the interoceptive, that is, homeostatic state of the body and the organism in general.

Paulus speaks of an 'interoceptive valuation' that describes the following: by providing interoceptive input to regions implicated in reward and decision making, the body and its actual vegetative state have a strong say in valuing the different options and thus to determine the degree of reward value that is assigned to the exteroceptive target stimulus. The value of the different options depends then not only on the predictability or probability and the reward magnitude of the exteroceptive stimulus but also on the actual interoceptive state of the body. This means that the interoceptive state and thus the bodily state can impact the degree of reward value and the consecutive set of preferences in subsequent decision making. 
By providing different interoceptive inputs, changes in bodily state can shift the valuing of the different options and hence set the preferences. These may, for instance, be abnormal, as Paulus (2007) argues, in addiction where the abnormal bodily vegetative state may lead subjects to assignment of abnormal reward values and ultimately to abnormal decision making.

Paulus uses the concept of "framing effects" to describe the impact of the bodily vegetative state on reward and value assignment (and subsequent decision making). This is more or less identical to what I mean by "context dependence" and more specifically "vegetative context dependence."

\section{Neuronal hypothesis IIB: difference-based coding and embodiment}

How can we further specify the dependence of reward and decision making on the interoceptive stimuli of the body and thus what Paulus describes as "framing effect", that is vegetative context-dependence? Let's recall briefly from the earlier sections in this chapter.

I suggested that the exteroceptive stimuli's natural and social statistics is encoded into neural activity. Thereby, the encoding of their statistical frequency distribution, the natural and social statistics, provided the common currency for the two different exteroceptive stimuli, that is, target and contextual stimuli, to be directly matched and compared with each other. This, in turn, was proposed to be possible only when presupposing difference-based coding (rather than stimulus-based coding) of the different stimuli's different spatial and temporal distributions.

What does this imply for intero-extero interaction? As in the case of exteroextero interaction, we are confronted with two different stimuli, interoceptive and exteroceptive stimuli. However, unlike in extero-extero interaction, the stimuli in intero-extero interaction have different origins. While in extero-extero interaction they both originate in the environment, they show different origins in intero-extero interaction namely body and environment. This raises the question of how stimuli from such different origins, body and environment, can directly interact with each other. For direct interaction to be possible, intero- and exteroceptive stimuli must share a common currency according to which both are processed.

How could such common currency look like? We often tacitly presuppose that the stimuli are encoded into neural activity on the basis of their origin. In our first example of reward, we tacitly assume that stimuli related to different persons like the own and the other person are encoded separately and thus in different ways. Analogously, we often tacitly assume that stimuli from the own body, i.e., interoceptive stimuli, are encoded separately from stimuli from the environment, i.e., exteroceptive stimuli. Such tacit assumption of origin-based coding (if one wants to say so) entails that the stimuli themselves and their different origin are encoded into neural activity thus entailing what I described above as stimulus-based coding.

The results, however, defy the tacit assumption of origin- and stimulus-based coding. Instead, the different stimuli including their different origins are processed in relation to each other, their difference or integral is computed and encoded into neural activity. This amounts to difference- rather than stimulus-based coding. The 
primary measure or metric according to which the brain encodes its neural activity is thus not the single stimulus itself and its origin but rather the differences between different stimuli across and independent of their different origins in own versus other person or body versus environment.

If exteroceptive stimuli are encoded in difference or relation to interoceptive stimuli, any kind of reward related to the former involves the own body and its interoceptive stimuli. This means that reward is embodied as one may want to describe it in conceptual terms. Briefly the concept of embodiment refers to the inclusion of the own body in the brain's neural processing and its higher-order cognitive processing. The concept of embodiment often refers to the involvement of sensorimotor functions as related to the body in higher-order cognitive functions like self or consciousness. Others like Damasio (1999) and Panksepp (1998) (see also Craig 2010; Critchley et al. 2004) also refer to the involvement of the body's vegetative, i.e., interoceptive, functions in the concept of embodiment.

I now postulate that the encoding of the difference or integral between intero- and exteroceptive stimuli into the brain's neural activity makes the involvement of body necessary, that is unavoidable, so that embodiment occur by default. Differencebased coding may thus be considered a necessary condition of embodiment that, taken in the sense of the involvement of the body, mirrors well what I described as "vegetative context-dependence" and what Paulus referred to as "framing effects".

\section{Neuronal hypothesis IIC: vegetative-context-dependence as non-instrumental context-dependence}

What exactly do I mean by vegetative context-dependence? The behavioral examples clearly indicate that the interoceptive stimuli can directly impact the exteroceptive stimuli which in turn changes the degree of reward. There is thus variation in all three, interoceptive stimuli, exteroceptive stimuli, and degree of reward. This presupposes instrumental context-dependence where the interoceptive stimuli impact the reward via changing the exteroceptive stimuli.

How about noninstrumental dependence? What Paulus describes as "framing effects" seems to close to noninstrumental dependence. The assignment of reward to exteroceptive stimuli takes place within the framework of and is thus constrained by the body's interoceptive stimuli. Due to their direct anatomical connections, the interoceptive stimuli can directly impact the neural activity in the reward system. Even if the exteroceptive stimuli remain the same and thus constant, our assignment of reward and the underlying reward-related neural activity may nevertheless change due to variation in the interoceptive input. This is possible only by assuming noninstrumental dependence of reward on interoceptive stimuli (see also Table 1).

In sum, both examples reflect noninstrumental dependence of the brain's neural activity during reward on its respective social and vegetative context. This is only possible if the brain encodes into its neural activity the relation and thus the difference between different stimuli. That amounts to what is described as difference-based coding as the brain's general encoding strategy as distinguished from stimulus-based coding (see Northoff 2013a for empirical details). 


\section{Neuronal findings IIIA: cognitive relationship between self and others}

How do we come to and make the assumption of attributing mental states and thus self and mind to other people? Philosophy has long relied on what is called the 'inference by analogy'. What is the 'inference by analogy'? The 'inference of analogy' goes like this. We observe another person A to show the behavior of type $\mathrm{X}$. And we know that in our own case the same behavior, e.g. type X, goes along with the mental state type M. Since our own behavior and the ones of the person A are similar, i.e., behavior of type of $\mathrm{X}$, we assume the other person A to show the same mental state type $\mathrm{M}$ we experience.

We thus infer from the analogy of behavior between us and the other person and our own associated mental states to the mental state of the latter. Hence, by indirect inference and analogy via our own case, we claim to obtain knowledge of the other person's mental state. How can we make such inference? Very simple. We may make it on the basis of our own mental states and their associated behavior. And what we do may also hold for the other person who in the same attributes mental states to us by inferring them from the comparison between our behavior they observe and their own mental states.

Why do we make such inference? Because it seems to be the most easiest and best way for us to explain the others' behavior. Only by assuming and inferring that you show mental states, I can explain your behavior. In other terms, your behavior of for instance taking the left street rather than the right one must originate in some kind of mental states that provides you with knowledge about the direction I, who chose the right street, do apparently not possess. The assumption of mental states thus seems to be the best explanation for your behavior. The 'inference by analogy' may thus be considered an inference to the best possible explanation.

The inference by analogy describes intersubjectivity in a very cognitive and ultimately linguistic way when attributing mental states and a self to other persons. In that case there would be no direct context-dependence of the self on other persons and thus the social context. There would thus be only indirect or instrumental contextdependence with a self that is primarily by itself independent of its social context.

\section{Neuronal findings IIIB: social relationship between self and others}

There may though be a deeper level of intersubjectivity. We also feel the other persons' mental states as for instance when sharing the emotional pain the spouse experiences when her father died. Such sharing of feeling is described as empathy and sheds the light on a deeper pre-cognitive and pre-verbal dimension of intersubjectivity as it has been emphasized in especially phenomenological philosophy [see for instance Zahavi (2005)].

Both, empathy and the attribution of mental states to another person are however slightly puzzling: despite that the fact that we do not experience the other's mental states and consciousness, we nevertheless either share them (as in empathy) or infer them (as in the inference by analogy). We have no direct access to other persons' 
experience of a self and its mental states in first-person perspective and nevertheless share their mental states and assume that they have a self.

How is such sharing of mental states possible? This is the moment where we need to introduce yet another perspective. There is the first-person perspective that is tied to the self itself and its mental states, the experience or consciousness of objects, events, or persons in the environment. And there is the third-person perspective that allows us to observe the objects, events, or persons in the environment from the outside rather than from the inside as in the experience in first-person perspective. The picture however is not complete yet. The interaction between the different selves as well as the second-person perspective as sandwiched between first- and third-person perspective are missing here.

What is the Second-Person Perspective? The Second-Person Perspective has initially been associated in philosophy with the introspection of the own mental states. Rather than actually experiencing the own mental states in first-person perspective, the second-person perspective makes possible to reflect and introspect about the own mental states. That is for instance the case when we ask ourselves whether it is really true that I heard the voice from another person speaking out there in the environment (see also Schilbach et al. 2013).

The second-person perspective thus allows to put the contents as experienced in first-person perspective into a wider context, the context of the own self as it is related to the environment. In other terms, the second-person perspective makes possible to situate and integrate the purely intra-individual self with its first-person perspective into a social context thereby transforming it into an inter-individual self. One can thus say that the concept of self is here determined in a social way so that one can speak of a 'social self' (Schilbach et al. 2013; Pfeiffer et al. 2013).

How can we define the concept of the social self? The concept of the social self describes the linkage and integration of the self into the social context of other selves. This shifts the focus from the experience or consciousness in the first-person perspective of a sole self to the various kinds of interactions between different selves as associated with the second-person perspective. As we already indicated there may be different kinds of social interactions including pre-cognitive and more cognitive ones.

Most importantly, the concept of the "social self" indicates that the self occurs in a social context; the experience of the own self is intimately linked to the other and thus on the respective social context. The concept of the "social self" consecutively entails director better non-instrumental (rather than instrumental) context-dependence of the self which though, admittedly, needs to be further supported and detailed in future empirical studies. This assumption is tentatively supported by recent data as they shall be described in the following.

\section{Neuronal findings IIIC: non-instrumental context-dependence of the self on the social context}

How can we investigate the social nature of the self? Various studies have been conducted to investigate different kinds of interaction between different selves. Pfeifer et al. (2013) and Schilbach et al. (2013) distinguish two different 
methodological approaches. One can investigate social cognition, the cognition of other people's mind, from the outside and thus from an observer's point of view. Social cognition is here investigated in an "offline" mode. More recently such "offline" methodological strategy has been complemented by an "online" mode. Here the social interaction is no longer investigated from the "outside" but rather from the "inside" by taking the perspective or point of view of the interacting selves themselves (rather than the observer's point of view).

Besides conducting several studies, the same group now recently investigated the neural overlap between emotional processing, resting state activity, and socialcognitive processing (Schilbach et al. 2012). They conducted a meta-analysis including imaging studies from all three kinds of investigations, resting state, emotional, and social-cognitive. In a first step they analysed the regions implicated in each of the three tasks. This yielded significant recruitment of neural activity in especially the midline regions like the ventro and dorsomedial prefrontal cortex and the posterior cingulate cortex (bordering to the precuneus). In addition, neural activity in the temporo-parietal junction and the middle temporal gyrus was observed.

In a second step they overlaid the three tasks, emotional, social-cognitive, and resting state, in order to detect commonly underlying areas. This indeed revealed the midline regions, the dorsomedial prefrontal cortex and the posterior cingulate cortex, to be commonly shared among emotional and social-cognitive tasks and the resting state activity. Based on this neural overlap the authors conclude that there may be an intrinsically social dimension in our neural activity which may be essential for any subsequent consciousness of both our own self and other selves. If this holds, our self including our intra-individual experience of it are indeed directly dependent upon the social context thus entailing non-instrumental rather than instrumental context-dependence as indicated above.

\section{Psychiatric evidence IA: depression and the increased self-focus}

I so far describe three instances where the neural activity in the brain and mental features like reward and self is directly and thus non-instrumentally dependent upon the context whether it is social or vegetative context. I postulate that such contextdependence is mediated neuronally by difference-based coding. How can we now support this claim? One way is to gather some indirect support from psychiatric disorders like major depressive disorder (MDD). In a first step I want to describe some cultural difference in the symptoms of MDD which, in a second step, is followed by the description of neuronal changes. Since the previous section focused on the self in healthy subjects, I now will restrict my discussion of MDD on its alterations in the self.

Major depressive disorder (MDD) is a complex psychiatric disorder that, in addition to prominent affective changes, can be characterized by bodily symptoms, ruminations, and withdrawal from the environment. Each of these latter symptoms reflects an increased focus on the self, the so-called "increased self-focus" in MDD (Thase 2005; Northoff 2007; Grimm et al. 2009, 2011; Alcaro et al. 2010) and hence an abnormally "reduced environmental focus". 
The symptom of increased self-focus in depressed patients concerns the pathologically heightened awareness of one's self including the own body. This state is made more debilitating by the fact that such individuals are no longer absorbed by positive interpersonal interaction or ongoing positive environmental objects and events. Their increased focus of one's experience on the self and the own body can also be defined by an increased interoceptive awareness (IA), the ability to perceive changes in the own body (Craig 2002, 2004; Critchley et al. 2004; Critchley 2005). The increased IA is contrasted with reduced exteroceptive awareness (EA), the awareness of stimuli that is events or objects in the environment.

The abnormal balance between IA and EA of depressed patients is accompanied by an abnormal experience of predominantly negative emotions and attribution of negative characteristics to one's own self. This is conform with prominent theories of emotions, which state that bodily processes and their awareness are important in the process of constituting an emotion, such as the James-Lange theory (James 1884; Lange 1887) or the somatic marker hypothesis by Damasio (1999). Though self and emotions are closely intertwined. It is important to distinguish them from each other since they seem to be subserved by distinct neural mechanisms (see for instance Panksepp 1998; Damasio 1999).

\section{Psychiatric evidence IB: cultural differences in depressive symptoms}

Is depression and its symptoms dependent upon its respective context? If so one would expect for instance cultural differences in depressive symptoms. Are their cultural differences in MDD? MDD has been observed to occur across all geographical areas studied, although with transcultural differences in symptom presentation and prevalence estimates (Ballenger et al. 2001). Somatic symptoms, due to heightened IA in MDD, have often been regarded to occur more often in nonwesterners (Ryder et al. 2002; Arnault et al. 2006; Ryder et al. 2008), although questions as to this position are raised by the fact that somatisation is also often observed in Western cultures (Kirmayer 2001; Kirmayer and Groleau 2001). What is clear though is that the cognition and determination of the self as mediated culturally strongly impacts upon the symptoms and course of depression (see for instance Kirmayer 2001), thus requiring research that focuses specifically on cultural neuroscience and psychopathology (Choudhury and Kirmayer 2009).

What remains unclear though is whether these transcultural differences are related to depression itself, thus being a feature of MDD, or whether they are related to more basic transcultural difference that is already present in healthy subjects. This is even more important to consider given the fact that psychological studies (Markus and Kitayama 1991; Markus and Kitayama 2003) demonstrate differences in IA and EA and self-referential processing between Eastern and Western cultures (Ma-Kellams et al. 2012).

Such studies have suggested that Western cultures presuppose a more individualistic concept of self, a construct that has been called the 'independent self' (Markus and Kitayama 2003; Kitayama and Park 2010). In contrast, the concept of self in Eastern cultures is more socially oriented, yielding what is described as 
'interdependent self' (Markus and Kitayama 1991; Kitayama and Park 2010). Moreover, Eastern cultures seem to be less accurate in perceiving changes in the own internal physiological state while being more driven by contextual external than interoceptive cues (Ma-Kellams et al. 2012).

These points raise the question as to whether the transcultural differences in depression are a feature of MDD itself or whether they reflect a principal East-West difference in the definition of one's self being present already in healthy subjects. The study of depressive symptomatology has shown that Chinese patients endorsed a higher proportion of somatic symptoms and that Chinese participants also used more somatic words when describing emotional experience compared to Westerners (Yen et al. 2000). A growing body of research supports that somatization reflects a poor interoceptive awareness, i.e., such somatization suggests a culturally bound tendency to misperceive one's own internal states (Gardner et al. 1990; Aronson et al. 2001; Bogaerts et al. 2008). However, to date, little research has examined potential underlying neuronal mechanisms due to transcultural differences.

\section{Psychiatric evidence IC: neural differences underlying the cultural differences in depression}

Recent neuroimaging studies investigated IA and EA in healthy subjects and found especially the insular cortex as a sensitive structure involved in interoceptive processes, but also anterior midline structures like the ACC. However, only little research in the field of neuroimaging has been done focussing on MDD patients and IA. Own results (Wiebking et al. 2010) suggest that differences between healthy and depressed subjects can be found in this particular brain region, the insula.

However, the results need to take into account the resting state activity of the brain, which has been shown to be abnormally elevated in MDD (specifically in cortical midline structures, but also on the global level of brain activity). This suggests that altered neuronal activity in brain regions involved in interoceptive processing may not be specifically related to the ability to perceive bodily changes but rather to the abnormal resting state in the insula in MDD (Northoff et al. 2010, 2011). The origin of abnormal resting state activity in the insula in MDD during interoception thus remains unclear: It may reflect supposedly culture-invariant abnormalities in the resting state and/or the bodily self per se, or, instead, abnormal reaction to culture-variant contents such as, for instance, social contextual cues associated with the self.

Other neuroimaging studies investigated healthy subjects during reflection (i.e., evaluation or judgment) about the degree of personal relevance, or self-relatedness, of stimuli (words, faces, sounds) (see Gillihan and Farah 2005; Legrand and Ruby 2009 for reviews). Highly self-related stimuli (like one's own face) yielded the most pronounced neuronal activity changes (i.e., elevated signal increases or reduced signal decreases in fMRI) in various medial regions of cortical and subcortical structures; these included the ventro- and dorsomedial prefrontal cortex (VMPFC, DMPFC), the perigenual anterior and posterior cingulate cortex (PACC, PCC), the ventral striatum (VS), the dorsomedial thalamus (DMT), and the tectum and 
colliculi (Gillihan and Farah 2005; Legrand and Ruby 2009). Furthermore, the above mentioned psychological East-West differences in the determination of one's self have recently been shown to correspond to neuronal differences in anterior midline structures like VMPFC and DMPFC in particular (Zhu et al. 2007; Chiao et al. 2010; Ng et al. 2010; Wang et al. 2012).

Interestingly, the same cortical midline regions (the VMPFC and DMPFC) have also been shown to respond in an abnormal way (i.e., elevated signal increases or reduced signal decreases in $\mathrm{fMRI}$ ) in Western MDD patients during judgement of the degree of self-relatedness of emotional stimuli (Lemogne et al. 2009, 2010 see below for further studies from our group). It remains unclear, however, as to whether this is due to culture-invariant resting state and/or self-related processing or to a differential response towards contextual information such as culture. Investigation of transcultural differences in resting state activity in both healthy and MDD subjects remains to be investigated in the future. Moreover, it remains unclear whether transcultural resting state activity difference predispose the different concepts of self like independent versus interdependent self as they characterize eastern and western cultures.

What does the example of depression can tell us about the context-dependence of the brain's neural activity and our mental features like self? Depression can be characterized by abnormal shifts in the context-dependence: the brain's neural activity and its respective psychological functions seem to be no longer as strongly dependent upon the social context as it is reflected in the decreased environmentfocus. Conversely, the context-dependence upon the own body and the own self seems to be abnormally enhanced corresponding to what we described as increased self-focus and increased body-focus. The abnormal balance between social contextdependence on the one hand and body and self-focus on the other seem to be related to the brain's resting state in especially the cortical midline regions.

Are the cortical midline regions and their resting state activity thus central for mediating the balance between the different forms of context-dependence and ultimately context-dependence itself? We have to be careful, however. The midline regions may mediate the balance between the different forms of context-dependence like between body and environment. This balance and thus the midline's resting state activity are altered in MDD. In contrast, the encoding of differences, i.e., differencebased coding, may by itself still be preserved as such in MDD. That however remains to be shown in the future though it is preliminarily (and rather indirectly) supported by the cultural differences in MDD symptoms which would remain impossible in the case of stimulus-based coding replacing difference-based coding.

\section{Conclusion}

What do the findings of cultural, social, and vegetative context-dependence tell us about how to determine the concept of culture? Culture is manifest in what I here described as non-instrumental context-dependence, the direct dependence of the brain's neural activity on its respective cultural, social, and vegetative context (thus presupposing the concept of context in a wider sense). Culture in this sense may 
thus be considered one instance of context-dependence besides others like social and vegetative context. Due to its focus on a particular context, the cultural context, cultural neuroscience, together with social neuroscience, may take a leading role in revealing one of the most basic principles of our brain's functions, its contextdependence (Northoff 2013a, b). Most importantly, as argued here, such contextdependence is direct and thus non-instrumental and, even more important, extends beyond the specific instance of cultural contexts to all kinds of different contexts including vegetative, social, and mental contexts. The insight into the noninstrumental context-dependence of our brain's neural activity may thus not only provide an answer to what culture is namely non-instrumental context-dependence, but also to our brain's neural code, that is, difference-based coding as distinguished from stimulus-based coding (Northoff 2013a, b).

\section{References}

Alcaro, A., Panksepp, J., et al. (2010). Is subcortical-cortical midline activity in depression mediated by glutamate and GABA? A cross-species translational approach. Neuroscience and Biobehavioral Reviews, 34(4), 592-605.

Arnault, D. S., Sakamoto, S., et al. (2006). Somatic and depressive symptoms in female Japanese and American students: A preliminary investigation. Transcultural Psychiatry, 43(2), 275-286.

Aronson, K. R., Barrett, L. F., et al. (2001). Feeling your body or feeling badly: Evidence for the limited validity of the Somatosensory Amplification Scale as an index of somatic sensitivity. Journal of Psychosomatic Research, 51(1), 387-394.

Ballenger, J. C., Davidson, J. R., et al. (2001). Consensus statement on transcultural issues in depression and anxiety from the International Consensus Group on Depression and Anxiety. Journal of Clinical Psychiatry, 62(Suppl 13), 47-55.

Bogaerts, K., Millen, A., et al. (2008). High symptom reporters are less interoceptively accurate in a symptom-related context. Journal of Psychosomatic Research, 65(5), 417-424.

Chiao, J. Y., Harada, T., et al. (2010). Dynamic cultural influences on neural representations of the self. Journal of Cognitive Neuroscience, 22(1), 1-11.

Choudhury, S., \& Kirmayer, L. J. (2009). Cultural neuroscience and psychopathology: Prospects for cultural psychiatry. Progress in Brain Research, 178, 263-283.

Craig, A. D. (2002). How do you feel? Interoception: The sense of the physiological condition of the body. Nature Reviews Neuroscience, 3(8), 655-666.

Craig, A. D. (2003). Interoception: The sense of the physiological condition of the body. Current Opinion in Neurobiology, 13(4), 500-505. doi:10.1016/S0959-4388(03)0090-4.

Craig, A. D. (2004). Human feelings: Why are some more aware than others? Trends in Cognitive Sciences, 8(6), 239-241.

Craig, A. D. (2005). Forebrain emotional asymmetry: A neuroanatomical basis? Trends in Cognitive Sciences, 9(12), 566-571. doi:10.1016/j.tics.2005.10.005.

Craig, A. D. (2010). The sentient self. Brain Structure and Function, 214(5-6), 563-577. doi:10.1007/ s00429-010-0248-y.

Critchley, H. D. (2005). Neural mechanisms of autonomic, affective, and cognitive integration. The Journal of Comparative Neurology, 493(1), 154-166.

Critchley, H. D., Wiens, S., et al. (2004). Neural systems supporting interoceptive awareness. Nature Neuroscience, 7(2), 189-195.

Damasio, A. R. (1999). The feeling of what happens: Body and emotion in the making of consciousness. New York: Harcourt Brace.

Engelmann, J. B., \& Hein, G. (2013). Contextual and social influences on valuation and choice. Progress in Brain Research, 202, 215-237. doi:10.1016/B978-0-444-62604-2.00013-7. 
Fehr, E., \& Camerer, C. F. (2007). Social neuroeconomics: The neural circuitry of social preferences. Trends in Cognitive Sciences, 11(10), 419-427.

Fiorillo, C. D., Newsome, W. T., \& Schultz, W. (2008). The temporal precision of reward prediction in dopamine neurons. Nature Neuroscience, 11(8), 966-973. doi:10.1038/nn.2159.

Fliessbach, K., Weber, B., Trautner, P., Dohmen, T., Sunde, U., Elger, C. E., et al. (2007). Social comparison affects reward-related brain activity in the human ventral striatum. Science, 318(5854), 1305-1308.

Friston, K. J. (1995). Neuronal transients. Proceedings. Biological Sciences/the Royal Society, 261(1362), 401-405. doi:10.1098/rspb.1995.0166.

Friston, K. J. (1997). Another neural code? Neuroimage, 5(3), 213-220.

Friston, K. J. (2000). The labile brain. I. Neuronal transients and nonlinear coupling. Philosophical Transactions of the Royal Society of London. Series B: Biological Sciences, 355(1394), 215-236.

Friston, K. J. (2010). The free-energy principle: A unified brain theory? Nature Reviews Neuroscience, 11(2), 127-138. doi:10.1038/nrn2787.

Gardner, R. M., Morrell, J. A, Jr., et al. (1990). Somatization tendencies and ability to detect internal body cues. Perceptual and Motor Skills, 71(2), 364-366.

Gillihan, S. J., \& Farah, M. J. (2005). Is self special? A critical review of evidence from experimental psychology and cognitive neuroscience. Psychological Bulletin, 131(1), 76-97.

Glimcher, P. W. (2011). Understanding dopamine and reinforcement learning: The dopamine reward prediction error hypothesis. Proceedings of the National Academy of Sciences USA, 108(42), 17568-17569. doi:10.1073/pnas.1114363108.

Grimm, S., Ernst, J., et al. (2009). Increased self-focus in major depressive disorder is related to neural abnormalities in subcortical-cortical midline structures. Human Brain Mapping, 30(8), 2617-2627.

Grimm, S., Ernst, J., et al. (2011). Reduced negative BOLD responses in the default-mode network and increased self-focus in depression. World Journal of Biological Psychiatry, 12(8), 627-637.

Han, S., \& Northoff, G. (2008). Culture-sensitive neural substrates of human cognition: A transcultural neuroimaging approach. Nature Reviews Neuroscience, 9(8), 646-654. doi:10.1038/nrn2456.

Han, S., Northoff, G., Vogeley, K., Wexler, B. E., Kitayama, S., \& Varnum, M. E. (2013). A cultural neuroscience approach to the biosocial nature of the human brain. Annual Review of Psychology, 64, 335-359. doi:10.1146/annurev-psych-071112-054629.

Hurely, S. (1998). Consciousness in action. Cambridge/Mass: Harvard University Press.

James, W. (1884). What is an emotion? Mind, 9, 185-205.

Kirk, U., Harvey, A., \& Montague, P. R. (2012). Domain expertise insulates against judgment bias by monetary favors through a modulation of ventromedial prefrontal cortex. Proceedings of the National Academy of Sciences USA, 108(25), 10332-10336. doi:10.1073/pnas.1205836109.

Kirmayer, L. J. (2001). Cultural variations in the clinical presentation of depression and anxiety: Implications for diagnosis and treatment. Journal of Clinical Psychiatry, 62(Suppl 13), 22-28. (discussion 29-30).

Kirmayer, L. J., \& Groleau, D. (2001). Affective disorders in cultural context. The Psychiatric Clinics of North America, 24(3), 465-478. vii.

Kitayama, S., \& Park, J. (2010). Cultural neuroscience of the self: Understanding the social grounding of the brain. Social Cognitive and Affective Neuroscience, 5(2-3), 111-129.

Knutson, B., \& Wimmer, G. E. (2007). Splitting the difference-how does the brain code reward episodes? Annals of the New York Academy of Sciences, 1104, 54-69. doi:10.1196/annals.1390.020.

Knutson, B., Wimmer, G. E., Rick, S., Hollon, N. G., Prelec, D., \& Loewenstein, G. (2008). Neural antecedents of the endowment effect. Neuron, 58(5), 814-822. doi:10.1016/j.neuron.2008.05.018.

Krebs, J. R., Kacelnik, A., \& Taylor, P. (1978). Test of optimal sampling by foraging great tits. Nature, $275,27-31$.

Lange, C. (1887). Ueber Gemuethsbewegungen. Eine Psycho-Physiologische Studie. Leipzig: Verlag von Theodor Thomas.

Legrand, D., \& Ruby, P. (2009). What is self-specific? Theoretical investigation and critical review of neuroimaging results. Psychological Review, 116(1), 252-282.

Lemogne, C., Bergouignan, L., et al. (2009). Cognitive avoidance of intrusive memories and autobiographical memory: Specificity, autonoetic consciousness, and self-perspective. Memory, 17(1), 1-7.

Lemogne, C., Mayberg, H., et al. (2010). Self-referential processing and the prefrontal cortex over the course of depression: A pilot study. Journal of Affective Disorders, 124(1-2), 196-201. 
Ma-Kellams, C., Blascovich, J., et al. (2012). Culture and the body: East-West differences in visceral perception. Journal of Personality and Social Psychology, 102(4), 718-728.

Markus, H. R., \& Kitayama, S. (1991). Culture and the Self: Implications for cognition, emotion, and motivation. Psychological Review, 98(2), 224-253.

Markus, H. R., \& Kitayama, S. (2003). Culture, self, and the reality of the social. Psychological Inquiry, 14(3/4), 277-283.

Menon, V. (2011). Large-scale brain networks and psychopathology: A unifying triple network model. Trends in Cognitive Sciences, 15(10), 483-506. doi:10.1016/j.tics.2011.08.003.

Montague, P. R. (2007a). The first wave. Trends in Cognitive Sciences, 11(10), 407-409. doi:10.1016/i. tics.2007.07.005.

Montague, P. R. (2007b). Neuroeconomics: A view from neuroscience. Functional Neurology, 22(4), 219-234.

Montague, P. R., \& Berns, G. S. (2002). Neural economics and the biological substrates of valuation. Neuron, 36(2), 265-284.

Montague, P. R., \& King-Casas, B. (2007). Efficient statistics, common currencies and the problem of reward-harvesting. Trends in Cognitive Sciences, 11(12), 514-519. doi:10.1016/j.tics.2007.10.002.

Montague, P. R., King-Casas, B., \& Cohen, J. D. (2006). Imaging valuation models in human choice. Annual Review of Neuroscience, 29, 417-448. Review.

Naqvi, N. H., \& Bechara, A. (2009). The hidden island of addiction: The insula. Trends in Neurosciences, 32(1), 56-67. doi:10.1016/j.tins.2008.09.009.

Naqvi, N. H., \& Bechara, A. (2010). The insula and drug addiction: An interoceptive view of pleasure, urges, and decision-making. Brain Structure and Function, 214(5-6), 435-450. doi:10.1007/s00429010-0268-7.

Ng, S., Han, S., et al. (2010). Dynamic bicultural brains: fMRI study of their flexible neural representation of self and significant others in response to culture primes. Asian Journal of Social Psychology, $13(2), 83-91$.

Northoff, G. (2007). Psychopathology and pathophysiology of the self in depression-neuropsychiatric hypothesis. Journal of Affective Disorders, 104(1-3), 1-14.

Northoff, G. (2013a). Unlocking the brain, vol 1: Coding. Oxford, NY: Oxford University Press.

Northoff, G. (2013b). Unlocking the brain, vol II: Consciousness. Oxford, NY: Oxford University Press.

Northoff, G., Qin, P., et al. (2010). Rest-stimulus interaction in the brain: A review. Trends in Neurosciences, 33(6), 277-284.

Northoff, G., Wiebking, C., et al. (2011). The 'resting-state hypothesis' of major depressive disorder-a translational subcortical-cortical framework for a system disorder. Neuroscience and Biobehavioral Reviews, 35(9), 1929-1945.

O'Doherty, J. P. (2004). Reward representations and reward-related learning in the human brain: Insights from neuroimaging. Current Opinion in Neurobiology, 14(6), 769-776.

O'Doherty, J. P. (2011). Contributions of the ventromedial prefrontal cortex to goal-directed action selection. Annals of the New York Academy of Sciences, 1239, 118-129. doi:10.1111/j.1749-6632. 2011.06290.x.

Panksepp, J. (1998). Affective neuroscience. New York: Oxford University Press.

Paulus, M. P. (2007). Decision-making dysfunctions in psychiatry-altered homeostatic processing? Science, 318(5850), 602-606. doi:10.1126/science.1142997.

Pfeiffer, U. J., Timmermans, B., Vogeley, K., Frith, C. D., \& Schilbach, L. (2013). Towards a neuroscience of social interaction. Frontiers in Human Neuroscience, 7, 22. doi:10.3389/fnhum. 2013.00022 .

Rangel, A. (2008). Consciousness meets neuroeconomics: What is the value of stimulus awareness in decision making? Neuron, 59(4), 525-527. doi:10.1016/j.neuron.2008.08.003.

Rolls, E. T. (2011). Chemosensory learning in the cortex. Frontiers in Systems Neuroscience, $5,78$.

Ryder, A. G., Bagby, R. M., et al. (2002). The overlap of depressive personality disorder and dysthymia: A categorical problem with a dimensional solution. Harvard Review of Psychiatry, 10(6), 337-352.

Ryder, A. G., Yang, J., et al. (2008). The cultural shaping of depression: Somatic symptoms in China, psychological symptoms in North America? Journal of Abnormal Psychology, 117(2), 300-313.

Schilbach, L., Bzdok, D., Timmermann, B., Fox, P. T., Laird, A. R., Vogeley, K., \& Eickhoff, S. B. (2012). Introspective minds: Using ALE meta-analyses to study commonalities in the neural correlates of emotional processing, social and unconstrained cognition. PLoS One, 7(2), e30920.

Schilbach, L., Timmermann, B., Reddy, V., Costall, A., Bente, G., \& Schlicht, T. (2013). Toward a second-person neuroscience. Behavioral and Brain Sciences (in press). 
Schultz, W. (2006). Behavioral theories and the neurophysiology of reward. Annual Review of Psychology, 57, 87-115. doi:10.1146/annurev.psych.56.091103.070229.

Schultz, W. (2007a). Behavioral dopamine signals. Trends in Neurosciences, 30(5), 203-210. doi:10. 1016/j.tins.2007.03.007.

Schultz, W. (2007b). Multiple dopamine functions at different time courses. Annual Review of Neuroscience, 30, 259-288. doi:10.1146/annurev.neuro.28.061604.135722.

Thase, E. (2005). Bipolar depression: Issues in diagnosis and treatment. Harvard Review of Psychiatry, 13, 257-271.

Wang, G., Mao, L., et al. (2012). Neural representations of close others in collectivistic brains. Social Cognitive and Affective Neuroscience, 7(2), 222-229.

Wiebking, C., Bauer, A., et al. (2010). Abnormal body perception and neural activity in the insula in depression: An fMRI study of the depressed "material me". The World Journal of Biological Psychiatry, 11(3), 538-549.

Yen, S., Robins, C. J., et al. (2000). A cross-cultural comparison of depressive symptom manifestation: China and the United States. Journal of Consulting and Clinical Psychology, 68(6), 993-999.

Zahavi, D. (2005). Subjectivity and selfhood: Investigating the first-person perspective. London: MIT Press.

Zhu, Y., Zhang, L., et al. (2007). Neural basis of cultural influence on self-representation. Neuroimage, 34(3), 1310-1316.

Zink, C. F., Pagnoni, G., Martin, M. E., Dhamala, M., \& Berns, G. S. (2003). Human striatal response to salient nonrewarding stimuli. The Journal of Neurosciences, 23(22), 8092-8097.

Zink, C. F., Pagnoni, G., Martin-Skurski, M. E., Chappelow, J. C., \& Berns, G. S. (2004). Human striatal responses to monetary reward depend on saliency. Neuron, 42(3), 509-517. 